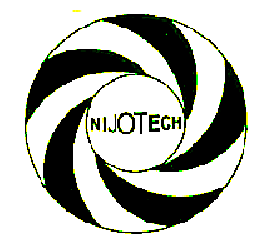

Nigerian Journal of Technology (NIJOTECH)

Vol. 33. No. 3, July 2014, pp. 258 - 267

Copyright@ Faculty of Engineering,

University of Nigeria, Nsukka, ISSN: $1115-8443$

www.nijotech.com

http://dx.doi.org/10.4314/njt.v33i3.3

\title{
STRUCTURAL AND ECONOMIC VIABILITY OF 2D/3D FINITE ELEMENT ANALYSIS OF CONICAL ARCHED ROOF TRUSS
}

\author{
J. C. Ezeokonkwo ${ }^{1,}$, C. U. Nwoji2, K. E. Ezugwu ${ }^{3}$ and N. C. Aneke 4 \\ 1,2,3,4 DePARTMENT OF Civil EnGINEERING, UNIVERSity OF NigERIA, NSUKKA, ENUGU STATE. NIGERIA \\ E-mail addresses: 1 josephat.ezeokonkwo@unn.edu.ng, 2clifford.nwoji@unn.edu.ng, \\ 3 ezugwukingsley@ymail.com,4anekenicholas@gmail.com
}

\begin{abstract}
Arch and conical roof trusses are employed when dealing with large span roof trusses. But a combination of the two geometrical shapes may lead to a better economical sections and a means of dealing with larger spans of roof truss that are not suitable for an arch or conical roof truss. Consequently, this paper examines the structural and economic viability of arched conical roof truss system based on 2D/3D finite element method analysis. Analysis of the results showed that truss members were subjected to higher axial forces in $2 D$ analysis than $3 D$ analysis, which will result to overdesign of the structural members and the attendant high construction cost. There are some variations observed in the axial forces for trusses located at edges of polygon when compared with the trusses located mid-point between two polygonal edges. This observation is quite significant in the sizes of the steel section properties of the members as well as in the fabrication and installation cost.
\end{abstract}

Keywords: arched conical trusses, hexagon, axial forces, steel section properties, economic viability

\section{INTRODUCTION}

Application of different geometrical shapes or combination of geometrical shapes to solve long span problems in roof design and construction by structural engineers and architects remains the only viable option. The use of either geometrical conical or arched roof shapes to overcome large span problem in many public buildings is wide spread. But the combination of the two most widely used shapes in long span roof buildings can lead to improved aesthetics, better space management, stability and economy. The behaviour of these shapes under the same stress conditions vary and the combination of the two will address their individual shortcomings in an environment where they exist together as a unit. Arched conical truss roof systems are not common neither are they used by architects nor engineers in the solution of long span problems, which is a major challenge in today's public buildings

Nevertheless, structural designers usually want to minimize the weight (or cost) and maximize the stiffness for given loads and available space. When a material is chosen, there are techniques available to optimize the geometry including topology, shape, and size [1]. On the other hand, for components of chosen geometry, most suitable materials can be systematically identified [2]. To optimize the performance and cost, the task of a designer is to determine the geometry of component(s) and material(s) for them under some functional requirements and constraints [3]. However, analysis and design of roof truss is based on fixing the geometry and then selecting the best material or determining the geometry for an arbitrarily selected material.

Prior to 1952 structural analysis was restricted to elements connected to only two points in space [4]. Structural engineers used the lattice analogy, as developed by Hrennikoff [5] and McHenry [6], to model membrane and plate bending parts of the structure. Courant [7] proposed setting up the solution of stresses in a variational form and then introduced the interpolation of shape functions over triangular sub regions making up the whole region as a method to obtain approximate numerical solutions. Levy [8] developed the flexibility or force method, and in 1953 [9] suggested the stiffness or displacement method as alternative method to analyze statically 
redundant aircraft structures. However, his equations were cumbersome to solve by hand, and thus the method became popular only with the advent of the high-speed digital computer.

The first treatment of two-dimensional elements was by Turner et al. in 1956 [10]. They derived stiffness matrices for truss elements, beam elements, and twodimensional triangular and rectangular elements in plane stress and outlined the procedure commonly known as the direct stiffness method for obtaining the total structure stiffness matrix. Along with the development of the high-speed digital computer, they developed the finite element stiffness equations expressed in matrix notation.

Extension of the finite element method to threedimensional problems with the development of a tetrahedral stiffness matrix was done by Martin [11]; Gallagher et al. [12] and Melosh [13]. Additional threedimensional elements were studied by Argyris [14]. The special case of axisymmetric solids was considered by Clough and Rashid [15] and Wilson [16].

Jiki [25] studied the effect of brace spacing on stress concentrations in welded square hollow section ' $\mathrm{K}$ ' joints and noted that high punching shear stresses from braces and bending of the chord seem to be critical when the spacing between braces exceed $50 \mathrm{~mm}$.

The method of structural analysis is divided into numerical solutions of differential equations for displacements or stress and matrix methods based on discrete-element idealization i.e. finite element method. Two complementary matrix methods of formulation of any structural problem are possible which are: the displacement method (stiffness method), where displacements are chosen as unknowns, and the force method (flexibility method), where forces are unknowns.

Division of structures into finite elements involves dividing the structure into members connected at joints, replacing curved arch by sufficient number of straight members so that the piecewise straight structure closely resembles the curved structure and continuum structures should be subdivided into triangular, rectangular or trapezoidal elements which are easy to solve.

However, this analogy could not be applied to nonrectangular areas. Ray Clough first faced this problem in the summers of 1952 and 1953 after joining the Boeing Summer Faculty Program and was asked to calculate the bending and torsional flexibility influence coefficients on low aspect wings. Static experimental results had been obtained for the sweptback box wing structure and they did not agree with the results produced by a structural analysis model using one-dimensional elements only [1]. The phrase finite element was introduced by Clough [17], when both triangular and rectangular elements were used for plane stress analysis.

After being developed and experimented with various applications for half a century, the finite element method increasingly evolved with the aid of computer programming, from being a lattice of single dimensional analysis to an enormous and advantageous application in solving complicated engineering programs from structural engineering to bioengineering $[18,19]$. Evolution of FEA is tied with the development in computer technology. With the enhancement in computer speed and storage capacity, FEA has become a very valuable engineering tool. NASA is credited with developing comprehensive FEA software in 1960's, known as NASTRAN. Rights of the software were purchased by McNeal Schwendler Corporation, who refined it and commercially marketed it under the name, MSC-NASTRAN. The first college course in FEA was offered in 1970. In the early 1970's, application of FEA was limited to large corporations, who can afford expensive mainframe computers. However, in 1980's, with the introduction of desktop computers, application of FEA became popular and indispensable engineering tool. In late 80 's, almost all the major FEA vendors introduced their software that can run on a PC [20]. These already developed theories and methods will be adopted in investigating the structural and economic viability of 2D/3D finite element method analysis of conical hexagonal arched roof truss.

The use of cone pitched roof has the advantage of minimising the effect of wind, because the cosine of angle of high pitched roof is small, thus it reduces the pulling effect of wind on the roof. It also reduces the effect of snow on roofs of buildings. An arch truss is a truss that includes the shape of an arch. These kinds of trusses are common in many different types of buildings. Traditional trusses are made with wood, but some truss types are also made of metal. An arch structure can be helpful in many different kinds of construction design. Typically, a bridge or other structure benefits from having a series of trusses arranged in an arch shape.

Onyia [26] noted that finite element model for the analysis of bridge decks offers more attractions than 
the chart-based method of distribution coefficients presently in use in many design offices.

\subsection{Formulation of finite element for truss system}

Finite elements employed in an analysis must be a true representation of geometry and mechanical properties of the structure. This classifies the elements into two categories: discrete elements- the geometry of these elements is simple and has a welldefined deflection equation; and continuous structure elements- have no well defined deflection or interpolation function, which is developed and approximated by using the theory of elasticity [20, 21]. For a truss element, distributed loads on a structure must be converted to the equivalent loads that are applied only at the nodes in any direction. The element resists only the axial component of the load while the perpendicular component to the element's axis merely causes free rotation at the joint $[20,21]$. The relationship between the local and global deflections is defined as

$$
\left(\begin{array}{l}
\delta_{1} \\
\delta_{2}
\end{array}\right)=\left(\begin{array}{llll}
C & S & 0 & 0 \\
0 & 0 & C & S
\end{array}\right)\left\{\begin{array}{l}
u_{1 x} \\
u_{1 y} \\
u_{2 x} \\
u_{2 y}
\end{array}\right\}
$$

Written in short form as

$(\delta)=(\mathrm{T})(\mathrm{u})$

Where $\delta_{1}$ and $\delta_{2}$ are local deflections

$\mathrm{T}=$ transformation matrix

$\mathrm{u}=\mathrm{u}_{1 \mathrm{x}}, \mathrm{u}_{1 \mathrm{y}}, \mathrm{u}_{2 \mathrm{x}}, \mathrm{u}_{2 \mathrm{y}}$ are global deflections

Furthermore, the local and global forces are related thus [20]:

$\{\mathrm{f}\}=(\mathrm{T})^{\mathrm{T}}\{\mathrm{R}\}$

Where $\{f\}=$ forces in global coordinates

$\{\mathrm{R}\}=$ forces in local coordinates

$(\mathrm{T})^{\mathrm{T}}=$ transpose of transformation matrix

Agarwal [20] further derive finite in the local coordinate system as

$\left|\begin{array}{l}R_{1} \\ R_{2}\end{array}\right|=\left[\begin{array}{cc}K & -K \\ -K & K\end{array}\right]\left[\begin{array}{l}\delta_{1} \\ \delta_{2}\end{array}\right]$

Where $\mathrm{K}=\mathrm{AE} / \mathrm{L}$

this implies that

$\mathrm{K}_{\mathrm{e}}=\left[\begin{array}{cc}\mathrm{AE} / \mathrm{L} & -\mathrm{AE} / \mathrm{L} \\ -\mathrm{AE} / \mathrm{L} & \mathrm{AE} / \mathrm{L}\end{array}\right]=$ stiffness matrix in local coordinates

Finally, the derived finite element equation in the global coordinate is as follows:

$\{f\}=[\mathrm{T}]^{\mathrm{T}}\left[\mathrm{K}_{\mathrm{e}}\right][\mathrm{T}]\{\mathrm{U}\}$

Implies that, $\{\mathrm{f}\}=\left[\mathrm{K}_{\mathrm{g}}\right]\{\mathrm{U}\}$

Where $\left[\mathrm{K}_{\mathrm{g}}\right]=\mathrm{T}^{\mathrm{T}}\left[\mathrm{K}_{\mathrm{e}}\right][\mathrm{T}]$

$$
\left[\mathrm{K}_{\mathrm{g}}\right]=\left[\begin{array}{ll}
\mathrm{C} & 0 \\
\mathrm{~S} & 0 \\
0 & \mathrm{C} \\
0 & \mathrm{~S}
\end{array}\right] \mathrm{AE} / \mathrm{L}\left[\begin{array}{cc}
1 & -1 \\
-1 & 1
\end{array}\right]\left[\begin{array}{llll}
\mathrm{C} & \mathrm{S} & 0 & 0 \\
0 & 0 & \mathrm{C} & \mathrm{S}
\end{array}\right]
$$

This gives,

$$
\left[\mathrm{K}_{\mathrm{g}}\right]=\mathrm{AE} / \mathrm{L}\left[\begin{array}{rrrr}
\mathrm{C}^{2} & \mathrm{CS} & -\mathrm{C}^{2} & -\mathrm{CS} \\
\mathrm{CS} & \mathrm{S}^{2} & -\mathrm{CS} & -\mathrm{S}^{2} \\
-\mathrm{C}^{2} & -\mathrm{CS} & \mathrm{C}^{2} & \mathrm{CS} \\
-\mathrm{CS} & -\mathrm{S}^{2} & \mathrm{CS} & \mathrm{S}^{2}
\end{array}\right]
$$

The above was derived in two dimensions.

Ananthasuresh [22] and Liu [23] noted that in an axially loaded bar, each node can only have one degree of freedom, i.e., deformation occurs along the axis while in trusses each node has two degrees of freedom, viz. deformations occurs in $X$ and $Y$ directions. There should be a constraint between the $X$ and $Y$ deformations such that the combined deformation is along the axis of the element and stiffness matrix for truss elements is derived as follows:

$\mathrm{q}=\left\{\begin{array}{l}\mathrm{q}_{1} \\ \mathrm{q}_{2}\end{array}\right\}=\left[\begin{array}{cccc}\mathrm{l} & \mathrm{m} & 0 & 0 \\ 0 & 0 & \mathrm{l} & \mathrm{m}\end{array}\right]\left\{\begin{array}{l}\mathrm{q}_{1} \\ \mathrm{q}_{2} \\ \mathrm{q}_{3} \\ \mathrm{q}_{4}\end{array}\right\}=\mathrm{L}_{\mathrm{q}}$

where

$\mathrm{l}=\cos \theta=\left(\mathrm{x}_{1}-\mathrm{x}_{2}\right) / \mathrm{L}_{\mathrm{e}}$ and $\mathrm{m}=\sin \theta=\left(\mathrm{y}_{1}-\mathrm{y}_{2}\right) / \mathrm{L}_{\mathrm{e}}$ are the direction cosines of the unit vector along the element.

The stiffness matrix for a truss element in its inclined orientation (2D) is

$\mathrm{K}_{\mathrm{e}}=\mathrm{L}^{\mathrm{T}} \mathrm{k}_{\mathrm{e}}{ }^{\prime} \mathrm{L}$

Where $\mathrm{K}_{\mathrm{e}}=\mathrm{A}_{\mathrm{e}} * \mathrm{E}_{\mathrm{e}} / \mathrm{L}_{\mathrm{e}}\left[\begin{array}{cc}1 & -1 \\ -1 & 1\end{array}\right]=$ element stiffness $\mathrm{L}=\left[\begin{array}{cccc}\mathrm{l} & \mathrm{m} & 0 & 0 \\ 0 & 0 & \mathrm{l} & \mathrm{m}\end{array}\right]=$ transformation matrix $\mathrm{L}^{\mathrm{T}}=\left[\begin{array}{cc}\mathrm{l} & 0 \\ \mathrm{~m} & 0 \\ 0 & \mathrm{l} \\ 0 & \mathrm{~m}\end{array}\right]=$ transpose of transformation matrix

$\mathrm{K}=\mathrm{AE} / \mathrm{L}\left[\begin{array}{cccc}\mathrm{l}^{2} & \mathrm{~lm} & -\mathrm{l}^{2} & \mathrm{~lm} \\ \mathrm{~lm} & \mathrm{~m}^{2} & -\mathrm{lm} & -\mathrm{m}^{2} \\ -\mathrm{l}^{2} & -\mathrm{lm} & \mathrm{l}^{2} & \mathrm{~lm} \\ -\mathrm{lm} & -\mathrm{m}^{2} & \mathrm{~lm} & \mathrm{~m}^{2}\end{array}\right]$

For any truss system, a node has two degrees of freedom. Therefore, for a truss system with $n$ numbers of nodes, the degree of freedom is $2 \mathrm{n}$ and the matrix is of order $2 \mathrm{nx} 2 \mathrm{n}$. Ananthasuresh [22] discussed the use of MATLAB script for FEM analysis of trusses and noted that MATLAB script requires four input files, which include geometric, physical and material property and force information about the truss to be analyzed. In 3D, a truss element is simply a two force member and has 
$\lambda=\left[\begin{array}{cccccc}\mathrm{l} & \mathrm{m} & \mathrm{n} & 0 & 0 & 0 \\ 0 & 0 & 0 & \mathrm{l} & \mathrm{m} & \mathrm{n}\end{array}\right]$

The element stiffness in global coordinates can be obtained using the formula

$[\mathrm{K}]=[\lambda]^{\mathrm{T}}\left[\mathrm{K}^{\prime}\right][\lambda]$

Substituting the above gives

$[\mathrm{K}]=\mathrm{A}_{\mathrm{e}} \mathrm{E}_{\mathrm{e}} / \mathrm{L}_{\mathrm{e}}\left[\begin{array}{cccccc}\mathrm{l}^{2} & \mathrm{~lm} & \mathrm{ln} & -\mathrm{l}^{2} & -\mathrm{lm} & -\mathrm{ln} \\ \mathrm{lm} & \mathrm{m}^{2} & \mathrm{mn} & -\mathrm{lm} & -\mathrm{m}^{2} & -\mathrm{mn} \\ \mathrm{ln} & \mathrm{mn} & \mathrm{n}^{2} & -\mathrm{ln} & -\mathrm{mn} & -\mathrm{n}^{2} \\ -\mathrm{l}^{2} & -\mathrm{lm} & -\mathrm{ln} & \mathrm{l}^{2} & \mathrm{~lm} & \mathrm{ln} \\ -\mathrm{lm} & -\mathrm{m}^{2} & -\mathrm{mn} & \mathrm{lm} & \mathrm{m}^{2} & \mathrm{mn} \\ -\mathrm{ln} & -\mathrm{mn} & -\mathrm{n}^{2} & \mathrm{ln} & \mathrm{mn} & \mathrm{n}^{2}\end{array}\right]$

The elements vector load $\mathrm{f}$ in local coordinates can be transformed into the global coordinates using

$\{f\}=[\lambda]^{\mathrm{T}}\left\{\mathrm{f}^{\prime}\right\}\{\lambda\}$

Roylance [24] stated that a very complicated stress problems can be solved using FEA, but the disadvantages of computer solutions must always be kept in mind. They do not necessarily reveal how the stresses are influenced by important problem on with as much closed form variables such as material properties and geometrical features, and errors in input data can produce widely incorrect results that may be overlooked by the analyst.

Theoretical modelling sharpens the designer's intuition; users of finite element codes should plan their strategy to supplement the computer simulation with as much closed form and experimental analysis as possible. Application of arched conical roof truss can be of benefit to structural engineers and architects dealing with large span structures. The present study is limited to conical arched hexagonal roof truss system. It can be extended to other polygonal cones. Thus this study examines the structural and economic viability of conical arched hexagonal roof truss using 2D/3D finite element method of analysis.

\subsection{Arched Conical Hexagonal Truss System}

The conical hexagonal arched roof truss shown in figure 1 is analyzed as a flat truss in $2 \mathrm{D}$ and $3 \mathrm{D}$. The hexagon has 6 equal sides spanning $8 \mathrm{~m}$ each. The roof is $3 \mathrm{~m}$ high while the king post has a length of $1.5 \mathrm{~m}$. With the above information, the co-ordinates of the arched roof truss are obtained for the two varying spans of $16 \mathrm{~m}$ and $13.86 \mathrm{~m}$ truss system. The tributary areas on the top nodes and tributary area at each connecting node position are shown in Table 1.

\subsection{Discretized Truss system}

The members defining the truss system has two nodes each, the first node and the second node whose co- ordinates have been defined earlier. The discretized 2D and 3D truss systems are shown in figures 2 and 3 respectively.

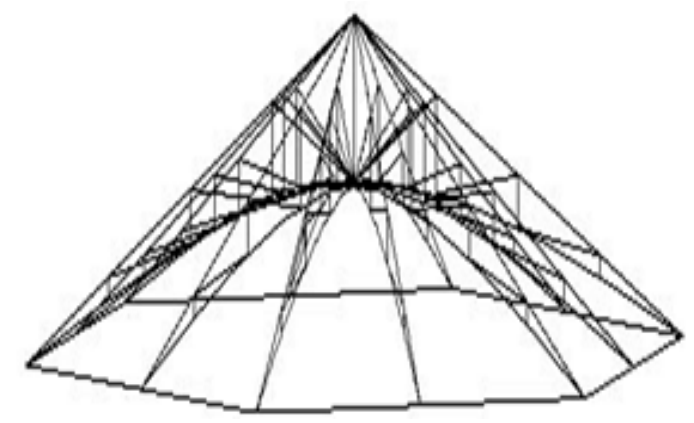

Figure 1: Conical hexagonal arched roof truss

\begin{tabular}{cccc}
\multicolumn{3}{c}{ Table 1: Tributary areas top nodes and connecting nodes. } \\
\hline S/N & $\begin{array}{c}\text { Top node } \\
\text { Area }\left(\mathrm{m}^{2}\right)\end{array}$ & Nodes & $\begin{array}{c}\text { Connecting } \\
\text { node }\left(\mathrm{m}^{2}\right)\end{array}$ \\
\hline 1 & 1.73 & $\mathrm{R}_{1}$ & 0.865 \\
2 & 5.19 & $\mathrm{R}_{2}$ & 1.2975 \\
3 & 8.65 & $\mathrm{R}_{3}$ & 2.307 \\
4 & 12.11 & $\mathrm{R}_{4}$ & 3.49 \\
pitch/apex & 0.865 & $\mathrm{R}_{5}$ & 2.048 \\
\hline
\end{tabular}

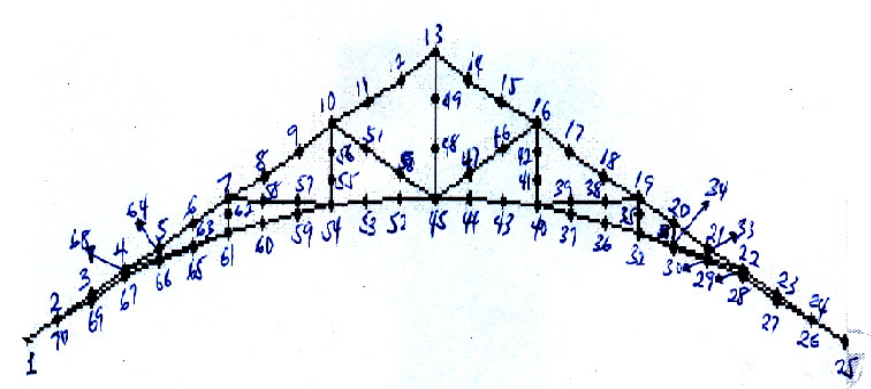

Figure 2. 2D Discretized truss system

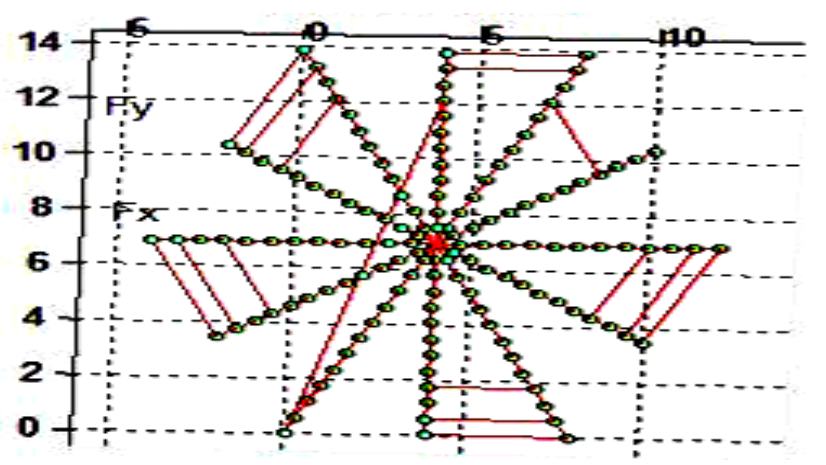

Figure 3: 3D Discretized truss system

\subsection{Matlab Models}

The MATLAB generated models are shown in Figures 4 and 5 for $2 \mathrm{D}$ and $3 \mathrm{D}$ respectively.

\section{ANALYSIS AND DESIGN}

\subsection{Analysis and results}

Due to the presence of numerous members in the truss system, the obtained results are analyzed such that each roof component is considered under the 
maximum tensile and compressive axial force in that component. Thus, Table 2 shows the results of analysis for 2D and 3D conditions for the two spans of 13.68 metres and 16.0 metres associated with conical hexagonal roof truss being considered. The class is the representative numbers assigned to discretized nodes and also used to identify the forces in elements connected at the nodes in the 2D and 3D analysis.

We shall take a look at the various type of axial forces on the members vis-à-vis tension $(+)$ and compression (-), with specific reference to 2D or 3D analysis. Tables 3 and 4 show the results of the comparisons of the 2D to 3D minimum and maximum axial forces in the two major spans for a conical hexagonal arched roof.

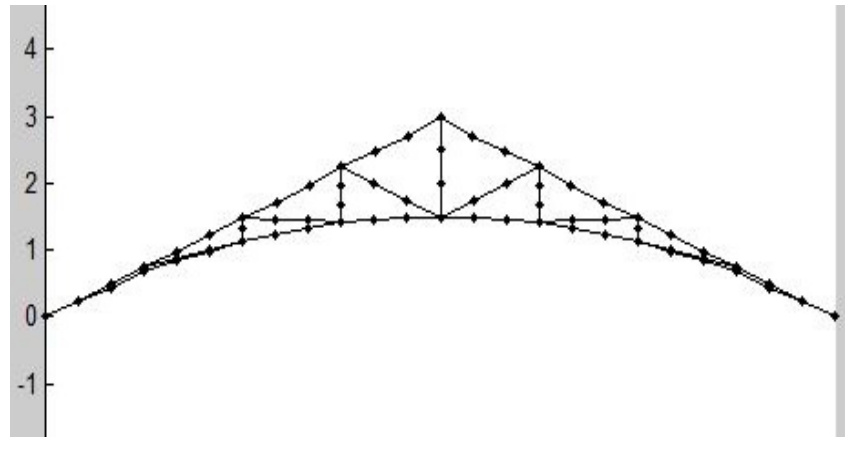

Figure 4: 2D Matlab model

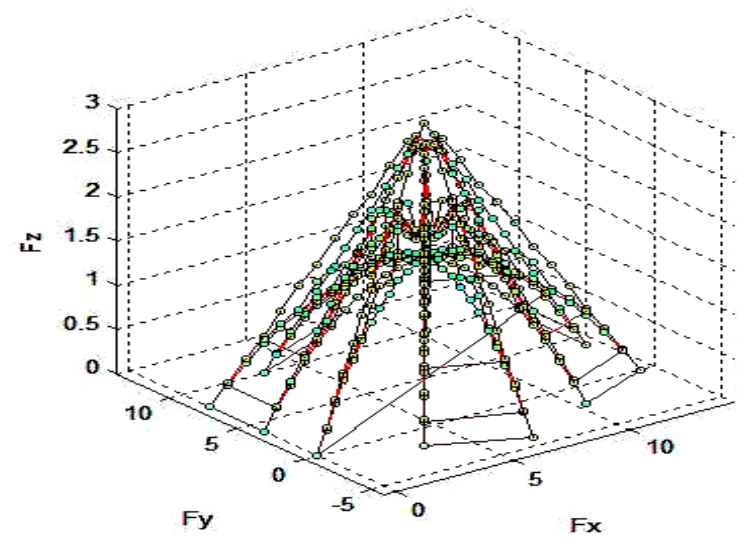

Figure 5: 3D Matlab model

chord members.

\begin{tabular}{|c|c|c|c|c|c|c|}
\hline \multirow{2}{*}{$\begin{array}{l}\text { Type of Truss } \\
\text { Member }\end{array}$} & \multicolumn{2}{|c|}{ Class } & \multicolumn{2}{|c|}{$\begin{array}{l}\text { Factored Load Combination (kN) } 13.68 \\
\text { Metres Span }\end{array}$} & \multicolumn{2}{|c|}{$\begin{array}{l}\text { Factored Load Combination (kN) } 16.0 \\
\text { Metres Span }\end{array}$} \\
\hline & $2 \mathrm{D}$ & $3 \mathrm{D}$ & $2 \mathrm{D}$ & 3D & $2 \mathrm{D}$ & 3D \\
\hline \multirow{24}{*}{ Top Chord } & 1 & 1 & 72.399 & -25.977 & 12.520 & -2.098 \\
\hline & 2 & 2 & 72.399 & -25.977 & 12.520 & -2.098 \\
\hline & 3 & 3 & 72.418 & -25.983 & 12.524 & -2.099 \\
\hline & 4 & 4 & 22.981 & 1.032 & 3.832 & 1.812 \\
\hline & 5 & 5 & 22.980 & 1.030 & 3.912 & 1.820 \\
\hline & 6 & 6 & 23.006 & 1.030 & 3.997 & 1.830 \\
\hline & 7 & 7 & 6.152 & 2.188 & 2.175 & -0.815 \\
\hline & 8 & 8 & 6.149 & 2.198 & 2.434 & -0.785 \\
\hline & 9 & 9 & 6.164 & 2.212 & 2.701 & -0.756 \\
\hline & 10 & 10 & -5.480 & 0.751 & 3.632 & -2.848 \\
\hline & 11 & 11 & -5.500 & 0.772 & 4.095 & -2.812 \\
\hline & 12 & 12 & -5.551 & 0.795 & 4.587 & -2.792 \\
\hline & 13 & 13 & -0.541 & 0.221 & 9.650 & 1.191 \\
\hline & 14 & 14 & -0.581 & 0.215 & 9.923 & 1.187 \\
\hline & 15 & 15 & -0.623 & 0.210 & 10.261 & 1.188 \\
\hline & 16 & 16 & -0.727 & 3.246 & 12.199 & 5.114 \\
\hline & 17 & 17 & -0.734 & 3.236 & 12.490 & 5.119 \\
\hline & 18 & 18 & -0.744 & 3.233 & 12.823 & 5.137 \\
\hline & 19 & 19 & -14.876 & 7.127 & 2.660 & 7.149 \\
\hline & 20 & 20 & -14.845 & 7.120 & 2.822 & 7.160 \\
\hline & 21 & 21 & -14.832 & 7.120 & 2.987 & 7.179 \\
\hline & 22 & 22 & -107.681 & 12.893 & -89.126 & -18.697 \\
\hline & 23 & 23 & -107.651 & 12.890 & -89.103 & -18.693 \\
\hline & 24 & 24 & -107.651 & 12.890 & -89.103 & -18.693 \\
\hline \multirow{3}{*}{ Bottom Chord } & 25 & 270 & 49.529 & -23.539 & 65.177 & 31.387 \\
\hline & 26 & 271 & 49.529 & -23.540 & 65.177 & 31.396 \\
\hline & 27 & 272 & 49.534 & -22.713 & 65.199 & 30.866 \\
\hline \multirow{2}{*}{ Web } & 28 & 328 & 4.878 & -2.512 & 2.724 & 6.264 \\
\hline & 29 & 327 & 3.819 & 1.119 & 0.343 & 2.138 \\
\hline Bottom Chord & 30 & 273 & 48.070 & -22.735 & 64.625 & 30.919 \\
\hline
\end{tabular}


STRUCTURAL AND ECONOMIC ViabiLiTY OF 2D/3D FEA OF ConicAl ARCHED Roof TRUSS, J. C. Ezeokonkwo, et al

\begin{tabular}{|c|c|c|c|c|c|c|}
\hline \multirow{4}{*}{$\begin{array}{l}\text { Type of Truss } \\
\text { Member }\end{array}$} & \multicolumn{2}{|c|}{ Class } & \multicolumn{2}{|c|}{$\begin{array}{l}\text { Factored Load Combination }(\mathrm{kN}) 13.68 \\
\text { Metres Span }\end{array}$} & \multicolumn{2}{|c|}{$\begin{array}{l}\text { Factored Load Combination (kN) } 16.0 \\
\text { Metres Span }\end{array}$} \\
\hline & $2 \mathrm{D}$ & $3 \mathrm{D}$ & $2 \mathrm{D}$ & $3 \mathrm{D}$ & $2 \mathrm{D}$ & $3 \mathrm{D}$ \\
\hline & 31 & 274 & 48.213 & -22.757 & 64.868 & 30.973 \\
\hline & 32 & 275 & 48.357 & -21.269 & 65.111 & -9.674 \\
\hline \multirow{5}{*}{ Web } & 33 & 326 & -96.101 & 1.091 & -97.485 & 1.719 \\
\hline & 34 & 325 & -95.930 & 1.064 & -97.151 & -40.688 \\
\hline & 35 & 324 & -95.759 & -2.168 & -96.818 & -40.622 \\
\hline & 36 & 323 & -1.558 & -2.044 & -0.792 & -40.557 \\
\hline & 37 & 322 & -2.151 & 0.746 & -2.764 & 0.617 \\
\hline \multirow{3}{*}{ Bottom Chord } & 38 & 276 & -47.023 & -21.305 & -31.322 & -9.596 \\
\hline & 39 & 277 & -46.812 & -21.341 & -30.890 & -9.518 \\
\hline & 40 & 278 & -46.603 & -20.531 & -30.458 & -17.545 \\
\hline \multirow{4}{*}{ Web } & 41 & 321 & -19.034 & 0.693 & -15.250 & 0.372 \\
\hline & 42 & 320 & -18.767 & 0.639 & -14.676 & -8.364 \\
\hline & 43 & 319 & -18.501 & -2.128 & -14.103 & -8.273 \\
\hline & 44 & 318 & -3.137 & -2.043 & -1.843 & -8.181 \\
\hline \multirow{3}{*}{ Bottom Chord } & 45 & 317 & -3.404 & -1.958 & -2.634 & -0.035 \\
\hline & 46 & 316 & -3.670 & 1.313 & -3.425 & -0.114 \\
\hline & 47 & 279 & -64.371 & -20.576 & -43.807 & -17.461 \\
\hline \multirow{11}{*}{ Web } & 48 & 280 & -64.132 & -20.621 & -43.311 & -17.381 \\
\hline & 49 & 281 & -63.893 & -11.105 & -42.825 & -22.654 \\
\hline & 50 & 315 & -3.892 & 1.256 & -1.420 & -0.192 \\
\hline & 51 & 314 & -3.657 & 1.199 & -1.171 & -2.200 \\
\hline & 52 & 313 & -3.423 & -2.636 & -0.921 & -2.143 \\
\hline & 53 & 310 & -9.386 & -2.305 & -0.283 & -2.305 \\
\hline & 54 & 311 & -9.422 & -2.270 & 0.231 & -2.270 \\
\hline & 55 & 312 & -9.457 & -2.086 & 0.745 & -2.086 \\
\hline & 56 & 309 & 9.357 & -2.665 & -3.848 & -2.340 \\
\hline & 57 & 308 & 9.563 & -2.695 & -3.238 & -0.363 \\
\hline & 58 & 307 & 9.770 & 0.370 & -2.627 & -0.282 \\
\hline \multirow{3}{*}{ Bottom Chord } & 59 & 282 & -75.394 & -11.154 & -39.623 & -22.586 \\
\hline & 60 & 283 & -75.153 & -11.202 & -39.189 & -22.525 \\
\hline & 61 & 284 & -74.913 & -3.560 & -38.765 & -21.292 \\
\hline \multirow{3}{*}{ Web } & 62 & 304 & -9.624 & -7.728 & -1.170 & -1.215 \\
\hline & 63 & 305 & -9.351 & 0.398 & 0.201 & -1.048 \\
\hline & 64 & 306 & -9.078 & 0.384 & 1.571 & -0.200 \\
\hline \multirow{3}{*}{ Bottom Chord } & 65 & 285 & -86.966 & -3.607 & -35.882 & -21.245 \\
\hline & 66 & 286 & -86.756 & -3.654 & -35.570 & -21.198 \\
\hline & 67 & 287 & -86.546 & 32.086 & -35.259 & -12.005 \\
\hline \multirow{5}{*}{ Web } & 68 & 303 & 11.409 & -7.778 & -2.644 & -1.382 \\
\hline & 69 & 302 & 11.677 & -7.829 & -2.087 & -1.317 \\
\hline & 70 & 301 & 11.946 & 0.903 & -1.529 & -1.239 \\
\hline & 71 & 300 & -9.689 & 1.000 & 0.170 & -1.162 \\
\hline & 72 & 299 & -10.291 & -35.529 & -1.565 & -1.088 \\
\hline \multirow{3}{*}{ Bottom Chord } & 73 & 288 & -129.208 & 32.050 & -38.371 & -11.981 \\
\hline & 74 & 289 & -129.066 & 32.014 & -38.225 & -11.958 \\
\hline & 75 & 290 & -128.924 & 33.178 & -38.079 & -12.151 \\
\hline \multirow{5}{*}{ Web } & 76 & 298 & 40.641 & -35.570 & 3.049 & -1.324 \\
\hline & 77 & 297 & 40.810 & -35.611 & 3.273 & -9.393 \\
\hline & 78 & 296 & 40.980 & 3.510 & 3.497 & -9.359 \\
\hline & 79 & 295 & -14.183 & 3.752 & -0.138 & -9.324 \\
\hline & 80 & 294 & -15.133 & -2.098 & -1.571 & -0.583 \\
\hline \multirow{3}{*}{ Bottom Chord } & 81 & 291 & -133.621 & 33.176 & -38.407 & -12.148 \\
\hline & 82 & 292 & -133.610 & 33.176 & -38.394 & -12.148 \\
\hline & 83 & 293 & -133.610 & 31.387 & -38.394 & -0.787 \\
\hline
\end{tabular}


STRUCTURAL AND ECONOMIC VIABILITY OF 2D/3D FEA OF CONICAL ARCHED ROOF TRUSS, J. C. Ezeokonkwo, et al

Table 3. Comparison of 2D to 3D Minimum and Maximum axial forces for 13.68 metres spans

\begin{tabular}{|c|c|c|c|c|c|c|c|c|c|}
\hline \multicolumn{10}{|c|}{$\begin{array}{l}\text { Factored Load Combination } \\
\text { Axial Forces }(\mathrm{kN})\end{array}$} \\
\hline & \multicolumn{4}{|c|}{ TENSION } & \multicolumn{4}{|c|}{ COMPRESSION } & \multirow[t]{2}{*}{ TYPE } \\
\hline & Class 2D & Force & Class 3D & Force & Class 2D & Force & Class3D & Force & \\
\hline & 3 & 72.418 & 22 & 12.893 & 22 & 107.681 & 3 & 25.983 & Top Chord \\
\hline Ratio & \multicolumn{4}{|c|}{5.617} & \multicolumn{4}{|c|}{4.144} & \\
\hline & 8 & 6.149 & 15 & 0.210 & 13 & 0.541 & $1 \& 2$ & 25.977 & Top Chord \\
\hline Ratio & \multicolumn{4}{|c|}{29.281} & \multicolumn{4}{|c|}{0.021} & \\
\hline & 27 & 49.534 & 290 & 33.178 & 291 & 133.621 & 271 & 23.540 & Bottom Chord \\
\hline Ratio & \multicolumn{4}{|c|}{1.493} & \multicolumn{4}{|c|}{5.676} & \\
\hline & 30 & 48.070 & 316 & 1.313 & 45 & 3.404 & 317 & 1.958 & Bottom Chord \\
\hline Ratio & \multicolumn{4}{|c|}{36.611} & \multicolumn{4}{|c|}{1.739} & \\
\hline & 78 & 40.980 & 295 & 3.752 & 33 & 96.101 & 297 & 35.611 & Web \\
\hline Ratio & \multicolumn{4}{|c|}{10.922} & \multicolumn{4}{|c|}{2.699} & \\
\hline & 29 & 3.819 & 307 & 0.370 & 36 & 1.558 & 318 & 2.043 & Web \\
\hline Ratio & \multicolumn{4}{|c|}{10.322} & \multicolumn{4}{|c|}{0.763} & \\
\hline
\end{tabular}

Table 4. Comparison of 2D to 3D Minimum and Maximum axial forces for 16.0 metres span

\begin{tabular}{|c|c|c|c|c|c|c|c|c|c|}
\hline \multicolumn{10}{|c|}{$\begin{array}{l}\text { Factored Load Combination } \\
\text { Axial Forces }(\mathrm{kN})\end{array}$} \\
\hline & \multicolumn{4}{|c|}{ TENSION } & \multicolumn{4}{|c|}{ COMPRESSION } & \multirow[t]{2}{*}{ TYPE } \\
\hline & Class 2D & Force & Class 3D & Force & Class 2D & Force & Class3D & Force & \\
\hline & 18 & 12.823 & 21 & 7.179 & 22 & 89.126 & 22 & 18.697 & Top Chord \\
\hline Ratio & \multicolumn{4}{|c|}{1.786} & \multicolumn{4}{|c|}{4.767} & \\
\hline & 7 & 2.175 & 14 & 1.187 & $23 \& 24$ & 89.103 & 9 & 0.756 & Top Chord \\
\hline Ratio & \multicolumn{4}{|c|}{1.832} & \multicolumn{4}{|c|}{117.861} & \\
\hline & 27 & 65.199 & 271 & 31.396 & 47 & 43.807 & 281 & 22.654 & Bottom Chord \\
\hline Ratio & \multicolumn{4}{|c|}{2.077} & \multicolumn{3}{|c|}{1.933} & & \\
\hline & 30 & 64.625 & 272 & 30.866 & 45 & 2.634 & 317 & 0.035 & Bottom Chord \\
\hline Ratio & \multicolumn{4}{|c|}{2.094} & \multicolumn{4}{|c|}{75.258} & \\
\hline & 78 & 3.497 & 328 & 6.264 & 33 & 97.485 & 325 & 40.688 & Web \\
\hline Ratio & \multicolumn{4}{|c|}{0.558} & \multicolumn{4}{|c|}{2.396} & \\
\hline & 71 & 0.170 & 321 & 0.372 & 79 & 0.138 & & 0.192 & Web \\
\hline Ratio & \multicolumn{4}{|c|}{0.457} & \multicolumn{4}{|c|}{0.719} & \\
\hline
\end{tabular}

\subsection{Pie Chart Analysis of Axial Forces}

The pie chart shown in figure 6 represents the magnitude of axial forces designed for in each method of analysis. Each method has two major axial forces classified as tension $(+)$ or compression $(-)$.

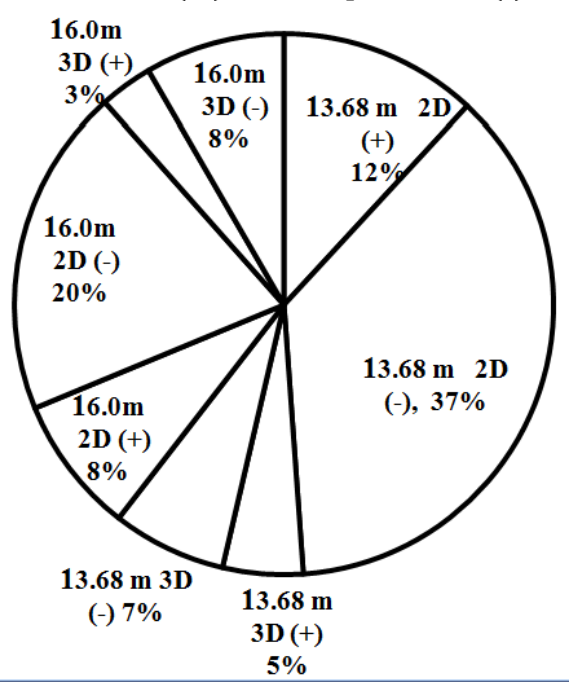

Figure 6: Summation of forces in each truss span

\subsection{Design of Steel Sections}

Steel design for the top, bottom and web members, based on the maximum and minimum tension or compression forces obtained in the 2D/3D analysis results shown in tables 3 and 4 for 13.86 metres and 16.0 metres spans respectively was undertaken. The design is based on the Steel Design Guide BS 5950-1: 2000 and Steel Designer's manual. Appropriate designed steel sections for the various members were selected and shown below in Table 5 .

Comparative cost analysis was undertaken based on the prevailing market prices of steel at Nsukka, Enugu State, Nigeria. This is based on the quantity (length in metres) of steel required for one $13.6 \mathrm{~m}$ and $16.0 \mathrm{~m}$ span roof truss respectively. Figure 7 shows the comparative cost analysis for $2 \mathrm{D}$ and $3 \mathrm{D}$ analysis. The costs of steel for the various members in 2D/3D analysis are also shown in Figure 5. 


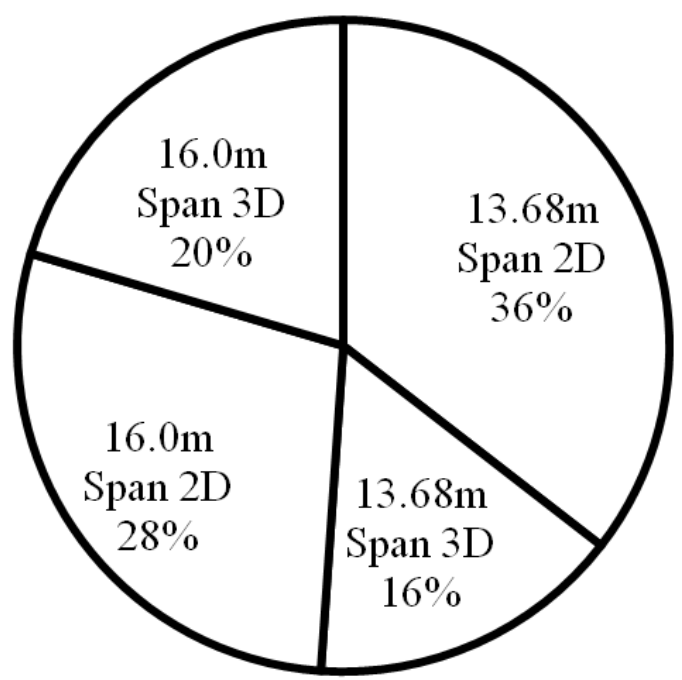

Figure 7: Cost analysis pie chart

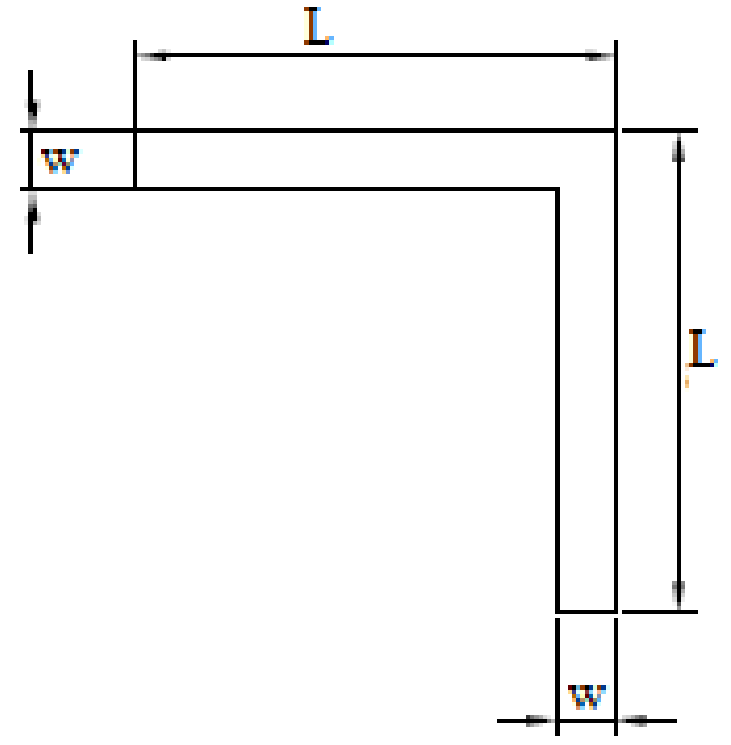

Figure 8: Pertaining to Table 5.

Table 5: Section properties based on 2-Dand 3-D analysis

\begin{tabular}{|c|c|c|c|c|c|c|c|}
\hline $\begin{array}{c}\text { Truss } \\
\text { Span (m) }\end{array}$ & Analysis & $\begin{array}{l}\text { Type of } \\
\text { Member }\end{array}$ & $\begin{array}{c}\mathrm{L} \\
(\mathrm{mm})\end{array}$ & $\begin{array}{l}\text { Thickness } \\
\mathrm{W}(\mathrm{mm})\end{array}$ & $\begin{array}{l}\text { Mass/Unit length } \\
(\mathrm{kg} / \mathrm{m})\end{array}$ & $\begin{array}{c}\text { Total } \\
\text { length }(\mathrm{m})\end{array}$ & $\operatorname{Cost}(\mathbb{N})$ \\
\hline \multirow{6}{*}{13.86} & \multirow{3}{*}{$2-\mathrm{D}$} & Top Chord & 50 & 5 & 3.77 & 15.27 & $21,178.19$ \\
\hline & & Bottom & 60 & 8 & 7.09 & 14.27 & $32,908.79$ \\
\hline & & Web & 40 & 5 & 2.97 & 14.83 & $20,567.93$ \\
\hline & \multirow{3}{*}{$3-D$} & Top & 25 & 3 & 1.12 & 15.27 & $11,295.06$ \\
\hline & & Bottom & 25 & 3 & 1.12 & 14.27 & $10,555.43$ \\
\hline & & Web & 30 & 3 & 1.36 & 14.83 & $10,969.65$ \\
\hline \multirow{6}{*}{16.00} & \multirow{3}{*}{$2-\mathrm{D}$} & Top & 40 & 4 & 2.42 & 17.09 & $18,961.89$ \\
\hline & & Bottom & 40 & 4 & 2.42 & 16.35 & $18,140.83$ \\
\hline & & Web & 40 & 5 & 2.97 & 16.42 & $22,773.18$ \\
\hline & \multirow{3}{*}{ 3-D } & Top & 25 & 3 & 1.12 & 17.09 & $12,641.42$ \\
\hline & & Bottom & 25 & 3 & 1.12 & 16.35 & $12,093.99$ \\
\hline & & Web & 30 & 4 & 1.78 & 16.42 & $18,218.58$ \\
\hline
\end{tabular}

\section{DISCUSSIONS}

It is obvious from Table 1 that the results obtained from 3D are far lesser than that from 2D analysis. As was shown in Table 3 for the 13.68 metres span truss, the results gotten from $2 \mathrm{D}$ is a multiple of the results from 3D ranging from 0.021 to 29.281 for top chord members; 1.493 to 36.611 for bottom chord members and 0.763 to 10.922 for the web members. The results depicted in Table 4 for 16.0 metres span truss shows that the results gotten from $2 \mathrm{D}$ is a multiple of the results from 3D ranging from 1.786 to 117.861 for top chord members; 1.933 to 3.347 for bottom chord members and 0.457 to 3.943 for the web members. Some of the multiples are very large for some members and would only lead to an overdesign of these structural steel members. Others have ratios less than unity such as minimum compressive forces in the top chord and web for the 13.68 metre span truss. Also the web for the 16.0 metre span truss has both the ratios for minimum tension and compression forces less than unity. Hence, for these members with the ratio of 2D force to 3D force less than unity, 2D analysis yields better economical sections.

The pie chart of figure 6 indicates the variations in the overall magnitude of the various axial force components resisted in the two span configurations of a conical hexagonal arched roof truss. It shows that the percentage of axial force generated through 2D analysis is $49.0 \%$ and $12.0 \%$ for 3D for 13.68 metres span truss and $28.0 \%$ for $2 \mathrm{D}$ and $11.0 \%$ for $3 \mathrm{D}$ in a 16.0 metres span truss. Analysis of the results of the various axial forces indicates that the edge truss in a conical hexagonal arched roof for the 16.0 metres span truss has less axial force than the 13.68 metres span truss located mid-way between the edges.

Table 5 and figure 7 show the section properties bases on 2D and 3D analysis and the corresponding cost implication. 2D based analysis gave higher steel 
section dimensions with a cost of \$74,654.53 (36\%); while 3D based analysis has lower steel section dimensions with a cost of $32,820.14$ (16\%) representing $43.962 \%$ of the cost of $2 \mathrm{D}$ analysis for 13.68 metres spans truss system. For the 16.0 metres span truss, 2D analysis also resulted in high steel section dimensions with a cost of $\$ 59,875.80$ (28\%) and 3D analysis gave lower steel section dimensions with a cost of 42959.76 (20\%) representing $71.738 \%$ of the cost of $2 \mathrm{D}$ analysis. It is evident that 2D based analysis would only lead to an overdesign of the structural steel members and a higher procurement cost.

\section{CONCLUSIONS}

This paper showed that a conical hexagonal arched roof truss analysis based on 2D, members were found to be subjected to high axial forces than in 3D. Proportionally, the differences in axial forces are quite high that employing 2D in analysis of such truss will always lead to overdesign of the structural members and the attendant higher procurement cost.

Finally, the results presented in this paper show that there are some variations observed in the axial forces for trusses located at edges of hexagon when compared with the trusses located mid-point between two hexagonal edges. This observation is quite significant in the sizes of the steel section members for 2D analysis for the two different spans considered, with the resultant higher procurement cost. But the 3D analysis for 13.68 metres and 16.0 metres spans roof truss systems, gave the same member sizes respectively for top chord, bottom chord and webs as shown in Table 5.

\section{REFERENCES}

[1] Bendsoe, M. P. and Sigmund, O., Structural Topology Optimization: Theory and Implementation, Springer verlag, Berlin, 2003.

[2] Ashby, M. F., Materials Selection in Mechanical Design, Butterworth-Heinemann, Boston, 1999.

[3] Ananthasuresh, G. K and Ashby, M. F "Concurrent Design and Material Selection for Trusses". Workshop: Optimal Design. Laboratoire de Mécanique des Solides Ecole Polytechnique Palaiseau France November 26-28, 2003.

[4] Clough, R. W and Wilson, E. L "Early Finite Element Research at Berkeley. Fifth U.S. National Conference on Computational Mechanics, Aug. 4-6, 1999.

[5] Hrennikoff, A. "Solution of Problems in Elasticity by the Framework Method", Journal of Applied Mechanics. 8, 1941, pp. 169-175.
[6] McHenry, D., "A Lattice Analogy for the Solution of Plane Stress Problems", Journal of Institution of Civil Engineers, 21 (2), 1943.

[7] Courant, R., "Variational methods for the solution of problems of equilibrium and vibrations," Bull. Amer. Math. Soc., 49 (1943), pp. 1-23.

[8] Levy, S., "Computation of Influence Coefficients for Aircraft structures with Discontinuities and Sweepback," J. Aeronaut. Sci., Vol. 14, No. 10, 1947, pp. 547-560.

[9] Levy, S., "Structural Analysis and Influence Coefficients for Delta Wings," J. Aeronaut. Sci., Vol. 20, No.7, July, 1953, pp. 449-454.

[10] Turner, M.J., Clough, R.W., Martin, H.C., and Topp, L.T. "Stiffness and Deflexion Analysis of Complex Structures", J. Aerospace Sci., Vol. 23, No. 9, 1956, pp. 805-823.

[11] Martín, H. C., "Plane Elastícity Problems and the Direct Stiffness Method," The Trend in Engineering, Vol. 13, Jan. 1961, pp. 5-19.

[12] Gallagher, R. H., Padlog, J., and Bijlaard, P. P., "Stress Analysis of Heated Complex Shapes," Journal of the American Rocket Society, Vol. 32, May 1962 pp. 700707.

[13] Melosh, R. J. "Basis for derivation for the direct stiffness method", AIAA Journal 1(7), 1963, pp 16311637.

[14] Argyris, J. H., "Recent Advances in Matrix Methods of Structural Analysis," Progress in Aeronautical Science, Vol. 4, Pergamon Press, New York, 1964.

[15] Clough, R. W., and Rashid, Y., "Finite Element Analysis of Axisymmetric Solids," Journal of the Engineering Mechanics Division, Vol. 91, Feb. 1965, pp. 71-85.

[16] Wilson, E. L. "Structural Analysis of Axisymmetric Solids," Journal of the American institute of Aeronautics and Astronautics, Vol. 3, No. 12, Dec. 1965, pp. 2269-2274.

[17] Clough, R. W., "The Finite Element Method in Plane Stress Analysis," Proceedings, American Society of Civil Engineers, 2nd Conference on Electronic Computation, Pittsburgh, PA, Sept. 1960. pp. 345378.

[18] ANSYS-386/ED Reference Manual, 1990.

[19] Logan, D. L. A First Course in the Finite Element Method. Detroit: Cengage-Engineering, 2006. Print.

[20] Agarwal, R. B Introduction to Finite Element Analysis. ME 273 Lecture Notes. P 1-7. http://www.engr.sjsu.edu/ragarwal/ME160/pdf/Ch apter\%201\%20-\%20Introduction.pdf Accessed July 2013.

[21] Reddy, J. N. "An Introduction to the Finite Element Method", Second Edition, McGraw-Hill, New York, 1993. 
[22] Ananthasuresh, G.K. "Finite Element MATLAB script for deflection and stress analysis of trusses", for MEAM 310 class 1997. http://www.mecheng. iisc.ernet.in/ suresh/me237/fea/Chapter6.pdf Accessed July 2013

[23] Liu, Y. Introduction to Finite Element Method, Lecture Notes , 1997. http://urbana.mie.uc.edu Lyliu/FEM-525/FEM-525.htm Accessed July 2013.

[24] Roylance D., "Finite Element Analysis", Department of Materials Science and Engineering, Massachusetts Institute of Technology, Cambridge, 2001. http://ocw.mit.edu/courses/materials-science-andengineering/3-11-mechanics-of-materials-fall1999/modules/fea.pdf Accessed July 2013.

[25] Jiki, P. N. "Prediction of Stress Concentration Factors in Unlapped Square Hollow "K" Joints by the Finite Element Method," Nigerian Journal of Technology, Vol. 25, No. 1, March 2006, pp 5-14.

[26] Onyia, M. E. "A Finite Element Model for the Analysis of Bridge Decks," Nigerian Journal of Technology, Vol. 27, No. 1, March 2008, pp 57-63 